\title{
ROLE OF RADIATION PROCESSING IN PRODUCTION OF HYDROGELS FOR MEDICAL APPLICATIONS
}

\author{
D. Darwis* \\ Center for Application of Isotope and Radiation Technology, BATAN \\ Jl Lebak Bulus Raya No. 49, Pasar Jumat, Jakarta Selatan, Indonesia \\ Received 27 February 2009; Received in revised form 27 May 2009; Accepted 16 June 2009
}

\begin{abstract}
ROLE OF RADIATION PROCESSING IN PRODUCTION OF HYDROGELS FOR MEDICAL APPLICATIONS. Recently, hydrophilic polymer gel (hydrogel) for application in medical fields has attracted much attention of researchers due to its unique properties which can resemble human living organs. Wound dressing, contact lenses and drug delivery system are among their applications in medical field. High energy radiation especially gamma ray and electron beam is often used for synthesis and modification of hydrogel. Through radiation crosslinking and or grafting process, hydrogel with specialty properties for specific application can be made. The advantage of radiation synthesized hydrogel over conventional methods is very pure products are obtained since the present of chemical initiators are not required; The preparation of sample does not require special sterile production rooms but still enables to obtain a sterile product; The irradiation process is easily controlled; Synthetis of new polymers and bulk or surface modification of commercial products can be accomplished with additional advantage of possibility of a concurrent sterilization. The future prospect of hydrogel seems to be in tissue engineering and diagnostic fields.
\end{abstract}

Keywords: Radiation processing, hydrogel, high energy radiation, biomedical

(c) 2009 Atom Indonesia. All rights reserved.

\section{INTRODUCTION}

Biomaterials are essentially materials which are used to repair, restore, or replace damaged or diseased tissue or to interface with the physiological environment. Biomaterials may be of natural origin, such as modified collagen, protein fibers (silk, wool, and hair), polysaccharides (starch, chitosan, cellulosic material), and treated tissues (pericardium, homo-, hetero-, and allograft), or synthetic biomaterials, such as metals, ceramics, or polymers [1].

Hydrogels are polymers characterized by hydrophilicity and insolubility in water. In water they swell to an equilibrium volume, but preserve their shape. The hydrophilicity is due to the presence of water solubilizing groups, such as --OH, -- $\mathrm{COOH},--\mathrm{CONH}_{2},--\mathrm{CONH}$, and $--\mathrm{SO}_{3} \mathrm{H}$. The insolubility and stability of the shape are due to the presence of

\footnotetext{
* Corresponding author.

E-mail address: darmawan_p3tir@batan.go.id (D. Darwis)
} 
three-dimensional network structures in the hydrogel [2-6]. Hydrogels are usually made of hydrophilic polymer molecules, which are crosslinked either by chemical bonds or other cohesion forces such as ionic interaction, hydrogen bonding, or hydrophobic interaction.

Hydrogels are elastic solids in the sense that there exists a remembered reference configuration to which the system returns even after being deformed for a very long time [6]. The term hydrogel implies that the material is already swollen in water. The dried hydrogel is called xerogel. During drying process water evaporates from the gel and the surface tension causes collapse of the gel body. Thus the gel shrinks to only a small fraction of its swollen size. There is no limit defining how much water a material has to absorb to become a hydrogel. In general, however, hydrogels are expected to absorb water at least $10-20 \%$ of their own weight. If a dried hydrogel imbibes at least 20 times it own weight of aqueous fluid while retaining its original shape, it is also called super absorbent. Due to their high water content, hydrogels usually have low mechanical strength.

In recent years, hydrogels (as one of the modern biomaterials) were developed for numerous applications in medicine, pharmacology and surgery [7-9]. Such products include hard materials for bone restoration, soft materials for tissue implants and artificial organ substitutes, contact lenses and various devices for delivery drugs, controlling drug release, healing wounds and many others. The common feature required for such applications is a certain high degree of tolerance of these materials in contact with tissue and body fluids. As a consequence, biomaterials have to be adjusted to very critical requirements differing widely for each particular application. In non-medical related application, hydrogels can be used for immobilization of microorganism for waste water treatment [10], chemo-mechanical energy conversion [11] and gel actuator [12].

The vast majority of work in the medical field can be traced back to Wichterle and his co-workers who first indicated the usefulness in biomedical applications of highly crosslinked polymer of 2-hydroxyethyl methacrylate (HEMA) [13]. The great advantages of this material over most other hydrophilic gels is its stability to varying conditions of $\mathrm{pH}$, temperature, and ionicity (osmotic concentration and salts) such as are commonly encountered in biomedical use.

Since the first report on the biomedical use of poly (HEMA), hydrogels with various properties have been prepared. Hydrogels can be made to respond (i.e. either shrink or expand) to changes in environmental conditions and the extent of the response can be controlled. Sometimes the volume change in response to the alteration in environmental conditions is so drastic and that phenomenon is called volume collapse or phase transition. The environmental conditions include $\mathrm{pH}$, temperature, electric field, ionic strength, salt type, solvent, external stress, light, and combinations of these. These unique properties have made hydrogels to be found in 
numerous applications, in pharmaceutical, agricultural, biomedical and human-oriented fields.

\section{SYNTHESIS OF HYDROGEL}

There are two methods that can be used to prepare hydrogel: radiation technique and conventional method. In the former method, hydrogel can be prepared by exposing water-soluble monomers or polymers to gamma radiation or fast electrons. In this technique, there is no need to add chemical initiators or crosslinking agents, which are mostly toxic materials $[7,8]$. In the second method, hydrogel is made by polymerizing of hydrophilic monomers in the presence of bi- or multifunctional crosslinking agents or by crosslinking of water soluble polymers using typical organic reactions which involve functional group of polymers [6].

\section{Radiation Crosslinking and Degradation}

One of the most striking observation made in the study of radiation effect in linear polymers is the fact that polymers either crosslink or degrade depending on chemical structure [14, 15]. Both processes take place simultaneously in most polymer systems. The one which predominates determines the net effect. Many years ago, Miller et al. [16] proposed

Table 1. Crosslinking vs chain scission for the polymer classes on exposure to ionizing radiation in vacuum

\begin{tabular}{|l|l|}
\hline Predominant crosslinking & Predominant degradation \\
\hline Polyethylene & Poly(isobutylene) \\
Polypropylene & Poly(vinylidene chloride) \\
PVC & PTFE \\
Chlorinated PE & Poly(methacrylonitrile) \\
Poly(acrylonitrile) & Poly(metacrylic acid) \\
Poly(acrylic acid) & Poly(metacrylate) \\
Poly(acrylate) & Poly(metacrylamide) \\
Poly(acrylamide) & Poly( $\alpha$-methylstyrene) \\
Polystyrene & Poly(ethylene terepthalate) \\
Natural rubber & Cellulosic \\
Polysiloxane & \\
Polyamide & \\
Poly(ethylene oxide) & Fully fluorinated thermoplastics \\
Polyester & Polychlorotrifluoroethylene \\
Poly(N-vinylpyrrolidone) & \\
Poly(vinyl alcohol) & \\
\hline
\end{tabular}


an empirical rule to predict the behavior of vinyl polymers during irradiation: the polymer tends to crosslink if each carbon atom of the main chain carries at least one hydrogen atom $\left(-\mathrm{CH}_{2}-\mathrm{CHR}-\right)_{\mathrm{n}}$, while degradation will predominantly occur in the polymer with tetrasubstituted carbon in the repeating unit like $\left(-\mathrm{CH}_{2}-\mathrm{CR}_{1} \mathrm{R}_{2}-\right)_{\mathrm{n}}$. Table 1 shows the classification of polymers into two groups according to the above mentioned criterion.

The net result of crosslinking is that the molecular weight of the polymer increases with increasing dose until a three dimensional network is formed where each polymer chain is linked to one other chain on average. The process of crosslinking or degradation schematically can be illustrated as follows.

Crosslinking reaction:

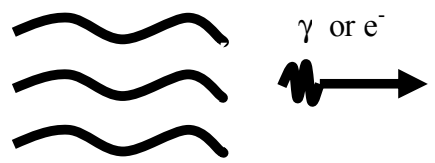

Polymer molecule chains

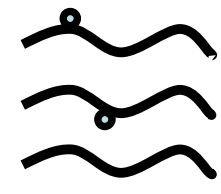

Polymer radicals

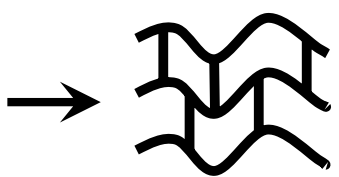

Crosslink

When scission predominates in an irradiated polymer, the molecular weight decreases as dose increases. The final product may be a low molecular weight liquid in some cases. The reaction can be indicated as follows.

Degradation reaction:

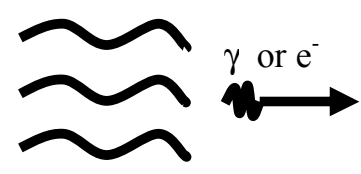

Polymer molecular chains

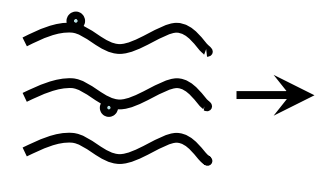

Polymer radicals

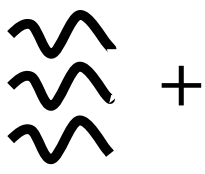

Low MW polymer

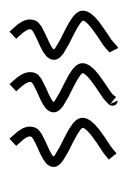

Low MW polymer

The application of ionizing radiation to produce hydrogels is mostly based on crosslinking reactions. However, hydrogels are also formed by copolymerization and subsequent crosslinking. Two types of ionizing radiation mainly used in preparing hydrogels are gamma rays generated from Cobalt-60 source and fast electron produced by accelerator $[3,17,18]$.

Polymer can be made by radiation crosslinking at various conditions both in solid state and in solution. However, crosslinking in solid state requires higher dose. In solution there are two general processes, i.e. direct effect and indirect effect. In the first case, the polymer molecules interact with ionizing radiation while in the latter case water radiolysis 
initiates the crosslinking by the formation of $\mathrm{OH} \bullet, \mathrm{H} \bullet$ radicals and $\mathrm{e}_{\mathrm{aq}}^{-}$ (solvated electron) [6].

Roughly, the mechanism of radiation crosslinking in the presence of water can be described as follows $[3,5]$.

Direct crosslinking:

Radiolysis of polymer

$$
\mathrm{RH} \longrightarrow \mathrm{R} \cdot+\mathbf{H}
$$

Recombination of polymer radicals

$$
\mathbf{R} \cdot+\mathbf{R} \cdot \longrightarrow \mathbf{R}-\mathbf{R}
$$

Indirect crosslinking:

Radiolysis of water

$$
\mathrm{H}_{2} \mathrm{O} \longrightarrow \mathrm{H} \cdot, \mathrm{OH} \bullet, \mathrm{e}^{-}{ }_{\text {aq }}
$$

Hydrogen abstraction

$$
\mathrm{RH}+\mathrm{OH} \longrightarrow \mathrm{R} \cdot+\mathrm{H}_{2} \mathrm{O}
$$

Recombination of polymer radicals

$$
\mathbf{R} \cdot+\mathbf{R} \cdot \longrightarrow \mathbf{R}-\mathbf{R}
$$

The detached studies on mechanism and kinetics of reactions for intermediate products of radiolysis of water with hydrophilic polymers such as: poly(vinyl pyrrolidone), poly(vinyl alcohol), poly(ethylene oxide), poly(vinyl methyl ether), poly(acrylic acid) have been recently performed by research groups of C. von Sontag (Germany) and J. M. Rosiak (Poland). They discussed kinetics and mechanism of decay of radicals as well as primary reactions leading to formation of intermoleculary crosslinked hydrogel and nano and microgels (intramoleculary crosslinking). Primary structure of macroradicals depends on chemical structure of the base unit of the main chain. Macro radical decay occurs via various reactions, among others, disproportionation and $\mathrm{H}$ transfer which play the dominant role $[19,20]$.

The relation between absorbed dose and sol remaining in the system has been established by Charlesby at the 1950s which is known as Charlesby-Pinner equation. In 1988, Rosiak elaborated this equation that can be used as a general equation of radiation crosslinking, which gives rise to Charlesby-Rosiak equation [19]: 
where:

$$
\mathrm{S}+\mathrm{S}^{1 / 2}=1 / 2 \psi+1 / 2(4-\psi)(\mathrm{Dv}+\mathrm{Dg})(\mathrm{Dv}+\mathrm{D})
$$

$\mathrm{S}=$ sol fraction (1-gel fraction); $\mathrm{Dg}=$ gelation dose; $\psi=$ relation between radiation yield of degradation and radiation yield of crosslinking.

$\mathrm{Dv}=$ virtual dose (compensation of none the most probable distribution of molecular weight and physical state of polymer e.g. crystallinity, present of crosslinkers, etc.).

Hydrogel chains are connected covalently in the form of three dimensional networks, so their molecular weights tend go to infinity. Such a network is presented schematically in Figure 1. The space between chains, represented by circle, is accessible for diffusion of solutes. In swollen state this space contains water or generally speaking molecules of solvent. The characteristic of this region, the mesh size, depends on the degree of crosslinking or in other words on the density of junction points. The higher the degree of crosslinking, the smaller amount of water can be absorbed. A simple control of the dose of irradiation makes it possible to prepare gels of any desired structure. In many cases the hydrogels for biomedical applications are produced by irradiation of monomers in their solutions [21].

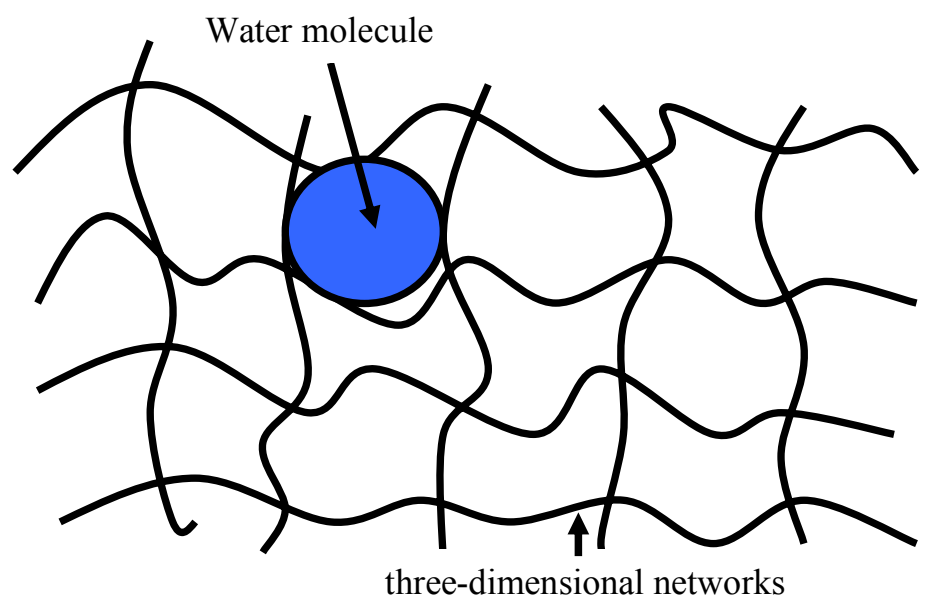

Figure 1. Illustration of three dimensional network crosslinking of hydrogel induced by radiation.

There are several advantages in using high energy radiation both from ${ }^{60} \mathrm{Co}$ source and from electron beam accelerator for production of hydrogels, i.e. very pure products are obtained since the presence of chemical initiator is not required; The preparation of sample does not require special sterile production rooms but still enables to obtain a sterile products; the irradiation process is easily controlled. Synthetis of new polymers and bulk or surface 
modification of commercial products can be prepared with additional advantage of possibility of a concurrent sterilization [7, 8, 17, 19, 20]

\section{Conventional Methods}

\section{Polymerization in the Presence of Crosslinking Agent}

Polymerization of water-soluble monomers in the presence of bi- or multifunctional crosslinking agents leads to chemical gels. Several monomers that are usually used for preparation of hydrogel are depicted in Table 2. The size of crosslinking agents can be varied widely. Not only low molecular weight crosslinking agents such as N-N-methylenebisacrylamide but also macromolecules such as protein can be used as crosslinking agents [6].

Table 2. Monomers used for production of hydrogels [6].

\begin{tabular}{|l|l|}
\hline \multicolumn{1}{|c|}{ Functional group of monomer } & \multicolumn{1}{c|}{ Example of monomer } \\
\hline Hydroxyl functional monomer & $\begin{array}{l}\text { 2 hydroxyethyl methacrylate (HEMA) } \\
\text { Hydroxypolyethoxy (10) allyl ether }\end{array}$ \\
\hline Vinyl functional monomer & $\begin{array}{l}\text { N-vinyl pyrrolidone } \\
\text { Vinyl acetate }\end{array}$ \\
\hline \multirow{2}{*}{ Acid functional monomer } & $\begin{array}{l}\text { Acrylic acid } \\
\text { Methacrylic acid } \\
\text { Beta-carboxyethyl acrylate }\end{array}$ \\
\hline Sulfonated monomer & $\begin{array}{l}\text { Sodium 1-allyloxy-2hydroxypropyl } \\
\text { sulfonate }\end{array}$ \\
\hline
\end{tabular}

Hydrogels based on methacrylic and acrylic esters can be made by copolymerizing monomers with a crosslinking agent such as ethylene dimethacrylate (EDMA). N-vinyl-2-pyrrolidone (NVP), a monomer which is soluble in water and many other solvents. Its strong interaction with water creates advantages and disadvantages with respect to hydrogel formation. Crosslinking agents such as N, N'-methylenebisacrylamide, divinylbenzene, and allyl methacrylate are often used. In hydrogels containing only NVP, high concentration of crosslinking agent (5-20\%) is needed [6].

\section{Crosslinking of Water Soluble Polymer}

Crosslinking of water-soluble polymers by addition of bi- or multifunctional reagent also results in chemical gel. A crosslinking agent has reactive groups at both ends and joins with two macromolecular components by covalent bonding. Polymers undergo the same reactions as their low molecular weight homologous, although the reaction rate and the maximum conversion in the reaction of polymer functional groups may differ significantly from those of the corresponding low molecular weight 
homologous [6]. The efficiency of crosslinking depends on the specificity toward particular groups to be functionalized. Thus the choice of crosslinking agents is critical to successful crosslinking.

\section{Crosslinking with Hydroxyl Groups}

A variety of crosslinking agents have been used to crosslink hydroxyl groups of polymer molecules, especially polysaccharides. Epoxides undergo ring opening reactions with nucleophiles including hydroxyl groups at alkaline $\mathrm{pH}$. Divinyl sulfone reacts with hydroxyl groups at alkaline $\mathrm{pH}$ value (e.g., $\mathrm{pH}$ 11). Imidazole can couple with amines to form stable carbamate (urethane) derivatives. Epichlorohydrin is commonly used to crosslink hydroxyl containing polysaccharide such as dextran, chitin, alginate, or hydroxyethylcellulose. Cyanuric chloride is also widely used for modifying hydroxyl groups. The chlorine atoms of the s-triazine series are very reactive toward nucleophiles including hydroxyl groups of carbohydrates.

\section{Crosslinking with Amine Groups}

The imidates and $\mathrm{N}$-succinimidyl derivatives are considered the most selective crosslinking agent for amino groups. Imidoesters readily react with amino groups with a high degree of specificity under mild conditions (pH 7-10). Albumin was crosslinked with bis(3-nitro-4-fluorophenyl)sulfone or (4,4'-difluoro-3,3'-dinitrodiphenylsulfone).

\section{Crosslinking with Carboxyl Groups}

There are relatively few crosslinking agents directed toward carboxyl groups. The epoxy group reacts, not only with amine groups, but also with carboxyl groups. Polyepoxydes were used to crosslink carboxyl groups of collagen, and bioprostheses such as porcine aortic leaflets or chinine carotid arteries. The treatment with polyepoxy compounds resulted in pronounced improvement in biocompatibility of the bioprostheses. In the presence of diamines, polymers containing carboxyl groups can be crosslinked by forming amide bond. Amide bonds between carboxyl and amino groups can be formed by many reagents, such as carbodiimide, Woodward's reagent K, ethylchloroformate, and carbodiimidazole. These crosslinking agents induce amide bond by removing atoms from the carboxyl and amine groups. 


\section{PROPERTIES OF HYDROGEL}

The are properties of hydrogel that can be described hereunder.

\section{Water Content}

The most important property of hydrogel is the ability to imbibe water. Thus the permeability of the membranes, their mechanical properties, their surface properties, and the resultant behavior at biological interfaces are all a direct consequence of the amount and nature of water held in this way. The amount of water absorbed at a given temperature is usually expressed as the equilibrium water content (EWC) $[2,5]$ :

$$
\operatorname{EWC}(\%)=\frac{W i}{W t} \times 100 \%
$$

where:

$\mathrm{Wi}=$ weight of water in the gel;

$\mathrm{Wt}=$ total weight of hydrated gel.

The EWC is governed by several factors. These include the nature of hydrophilic group present in the gel, the nature and density of crosslink and the external factors such as temperature, the tonicity (together with the nature of the constituent ions), and the $\mathrm{pH}$ of hydrating medium. It is therefore, the fact that the nature of hydrogels is dominated by the effect of water within the matrices that gives them their unique role in the field of biomedical applications. Water content of hydrogel also can be expressed as degree of swelling, DS, defined as gram of water in the swollen membrane per gram of dried gel.

\section{Crosslink Density}

The degree of crosslinking (crosslink density) in hydrogels can be determined by measuring either its equilibrium swelling or equilibrium elastic modulus [6, 19]. In the former case, the calculation is based on the equation:

$$
\mathrm{Ve} / \mathrm{Vo}=(0.5-\chi) /\left(\mathrm{v}_{1} \Phi o^{2 / 3} \mathrm{Q}^{5 / 3}\right)
$$

where:

$\mathrm{Ve} / \mathrm{Vo}=$ the effective network chain concentration or effective chain density (in the unit of $\mathrm{mol} / \mathrm{cm}^{3}$ ),

$\chi=$ Flory-Huggins interaction parameter, $\mathrm{v}_{1}$ is molar volume of water,

$\Phi_{0} \quad=$ degree of swelling,

$\mathrm{Q}=$ volume ratio of the swollen to the unswollen hydrogel. 
As it can be seen in Figure 2, DS of PVA membranes decreases with dose. This is due to the increasing of crosslink density as irradiation dose increases. In simplistic terms the water conveys a degree of surface hydrophilicity that is absent in more conventional polymers. DS also facilitates a degree of transport of water-soluble species that is difficult to achieve in non hydrogel materials.

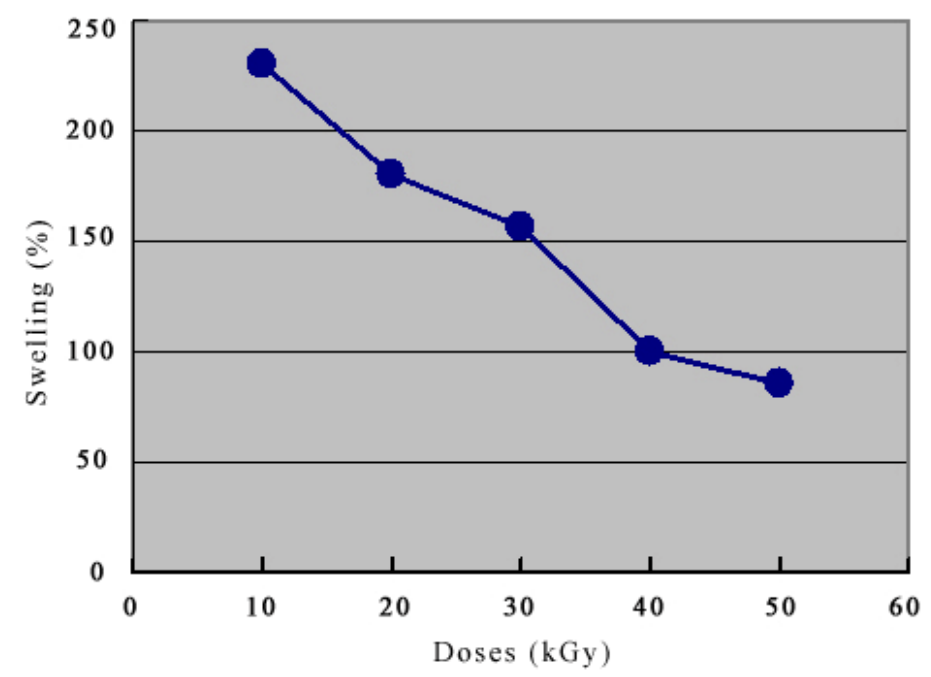

Figure 2. Swelling characteristic of PVA hydrogel at different irradiation doses [8].

\section{Gases Permeability}

Permeation in hydrogels has a particular importance in two applications: the permeation of oxygen through contact lenses and the permeation of drugs in hydrogel delivery systems. Oxygen permeability is of crucial importance for continuous wear contact lenses. The permeability of oxygen through hydrogel lenses is determined by water content because the diffusion of oxygen occurs in the water part of the gel. Thus, the oxygen permeability through hydrogel increases exponentially with increasing specific water content irrespective of the type of polymer performing the hydrogel network.

Swelling and permeation reflect the internal properties of hydrogel. Surface properties are critical for cell adhesion, thrombogenicity, and biocompatibility. Three surface properties are essential for blood compatibility: water content, ionic character and wettability of the surface. A high water content is generally considered favorable, but there are exceptions caused by other effects. 


\section{Mechanical Properties}

For a polymer to be used in biomedical devices, it must have the appropriate mechanical properties. Hydrogels, due to their high water content, show weak mechanical performance. Many attempts have been made to improve mechanical properties of hydrogel. Copolymerization of 2-hydroxyethyl methacrylate hydrogel with n-phenyl methacrylate, vynil propionate, and vynil acetate resulted in mechanical strength of about twice of the pure 2-hydroxyl methacrylate. Polyvinyl alcohol hydrogel with high tensile strength and heat resistance properties was found by combination of chemical and radiation crosslinking [22]. Grafting of hydrophilic monomer onto hydrophobic polymer by radiation technique also resulted in increases of the mechanical properties. Tensile strength of styrene butadiene rubber (SBR) graft vynil pyrrolidone (VP) is higher than that of its individual polymers [23].

\section{Biocompatibility}

Biocompatibility is another important parameter to be considered when a material will be used in biomedical. Biocompatibility defined as the ability of material to perform with an appropriate host response in a specific application. Therefore biocompatibility is concerned with all of the reactions that take place between a material and the body [24].

\section{MEDICAL APPLICATION OF HYDROGELS}

As already mentioned above, hydrogels are principally used in medicine. They are biomaterials, used in diagnostic or therapeutic devices and implants for short or long applications. The main applications of hydrogels as biomedical materials are:

\section{Contact Lenses}

Contac lenses are optical devices that are placed over cornea eye in such a manner that the lens remains on the eye surface throughout blinking. The main purpose of contact lenses is correction of vision deficiencies; in this application they are called cosmetic lenses. Contact lenses can also be used medically for the treatment of certain corneal diseases, in such cases they are called therapeutic, or bandage lenses [25]. The market of contact lenses is completely dominated by products on the base of polyHEMA. There is also a great number of various hydrogel compositions, which contain copolymers such as poly(vynil pyrrolidone), poly(vynil alcohol), poly(methacrylic acid), chitosan, and silicon. Copolymerization is mainly used to improve polyHEMA mechanical properties and increase its oxygen permeability [8]. 
Some contact lenses produced by radiation technology have been marketed in China [17].

Contact lenses can generally be classified as hard lenses, flexible lenses, and soft hydrogel lenses. In all three types there are lenses that are oxygen permeable and in hard and flexible types there are lenses that are hydrophobic. An essential difference among the three types is the manner in which they fit the eye. Hard lenses and hydrophobic flexible ones require a relatively thick tear film between their posterior surface and the cornea of the eye, which is a matter of considerable optical and physiological importance. Soft hydrogel lenses adhere closely to the cornea with a tear film of only capillary thickness between the lens and the cornea surface.

It is estimated that more than 10 million people have purchased poly(methyl methacrylate) contact lenses in the United States. However, more than half of these purchasers do not continue to wear the lenses because of discomfort and adjustment difficulties. The main problems encountered in the contact lenses (especially hard contact lenses) are oxygen permeability, wettability, mechanical properties (for hydrogel contact lens), protein adsorption, etc. Poly(methyl methacrylate) hard contact lenses are practically impermeable to oxygen. In order for a contact lens to be comfortable, one of the most important requirements is that its surface must be uniformly wet by the tear film at all times. Good water wettability is also required for good optical performance of lens and for its ability to remain clean of mucoid deposits in eyes. The corneal surface must be kept wet and oxygenated to remain transparent and healthy. Tear film wets the cornea, supplying the epithelium with oxygen from the air. If corneal is deprived of atmospheric oxygen, epithelium glycogen decreases as a result of anaerobic glycolysis. Hydrophobic flexible lenses manufactured from silicone rubber are highly permeable to oxygen and can maintain normal respiration on the cornea surface without tear exchange under the lens. The surface can be modified to make these lenses less hydrophobic, but such treatment reduces oxygen permeability.

According to Polse and Mandell [3], the minimum oxygen required by the epithelium of the cornea is about $3.5 \mu \mathrm{L}(\mathrm{STP}) /\left(\mathrm{cm}^{2} . \mathrm{h}\right)$. The cornea will swell if less than this amount of oxygen is available. The oxygen permeability of some contact lenses is shown in Table 3. 
Table 3. Oxygen permeability of some hard contact lenses and hydrogel soft contact lenses materials at room temperature [3].

\begin{tabular}{|l|c|}
\hline \multicolumn{1}{|c|}{ Contact lens } & $\begin{array}{c}\text { Oxygen permeability } \\
\boldsymbol{\mu} \mathbf{L}(\mathbf{S T P}) . \mathbf{c m} /\left(\mathbf{c m}^{2} \mathbf{. h . k P a}\right)\end{array}$ \\
\hline Hard contact lenses & 0.27 \\
\hline Poly(methyl methacrylate) & 64.8 \\
\hline Poly(4-methyl-1-pentene) & 10.4 \\
\hline Cellulose acetate butyrate & 13.0 \\
\hline Hydrogel soft contact lenses & 25.7 \\
\hline Poly(hydroxyethyl methacrylate) & 39.2 \\
\hline $\begin{array}{l}\text { Poly(hydroxyethylmethacrylate-co- } \\
\text { vinylpyrrolidone (hefilcon A) }\end{array}$ & 19.3 \\
\hline $\begin{array}{l}\text { Poly(hydroxyethylmethacrylate-co- } \\
\text { methacrylic acid-co-vinylpyrrolidone) }\end{array}$ & \\
\hline $\begin{array}{l}\text { Poly(hydroxypropylmethacrylate-co- } \\
\text { methylmethacrylate) (crofilcon A) }\end{array}$ & \\
\hline
\end{tabular}

The pioneering work of Wichterle and Lim with acrylic hydrogel revolutionized the contact lens industry. The first hydrogel contact lenses were made from slightly crosslinked poly(2-hydroxyethyl methacrylate), which is still commonly used for soft contact lenses. Hydrogels soft contact lenses can overcome some of the problems due to their high water content and good wettability. Poly(hydroxyethyl methacrylate) hydrogels equilibrate in water at room temperature to approximately $40 \%$ of its weight. This hydrogel contact lens was prepared from the mixture of 2-hydroxyetyl methacrylate monomer with other hydroxyalkyl methacrylate by using gamma radiation.

A contact lens material of about twice the tensile strength of pure 2-hydroxyethyl methacrylate hydrogel was obtained by copolymerizing this monomer with smaller amounts of n-phenyl methacrylate, vinyl propionate, and vynil acetate.

\section{Wound Dressings}

The development of synthetic occlusive wound dressing which is used for treatment of burns, blisters, fissures, herpes, and several other skin conditions, is currently a subject of great commercial interest.

Although there is no general consensus, an ideal wound dressing material should have several properties such as [13]: The material should be flexible, strong enough and does not give any pain when exchanging the dressing; it absorbs effectively the body fluids and prevents their loss, prevents contamination of the wound by microorganisms from outside, enables oxygen penetration; it adheres well to the wound but is stronger to 
the healthy skin and enables control of drug dosage; it is non antigenic, biocompatibility to the skin and blood; it is easy to sterilize, has good transparency.

Hydrogels posses many of the above properties to a certain degree and because of this they have been examined in various forms as wound dressing. The first commercial synthetic hydrogel wound dressing produced by radiation technique is Aqua-gel ${ }^{\circledR}$ [26]. It is claimed that this hydrogel is transparent, has over $90 \%$ of water content, enables of control drug dosage, offer good handling (i.e. easy placement and replacement without pain), and due to radiation processing it is fully sterile $[8,25]$. An example of Aqua-gel hydrogel is shown in Figure 3. Another hydrogel wound dressing based on poly(vinyl alcohol) prepared by electron beam radiation is nearly commercialized by Nichiban Company in Japan (Yoshii, private communication).

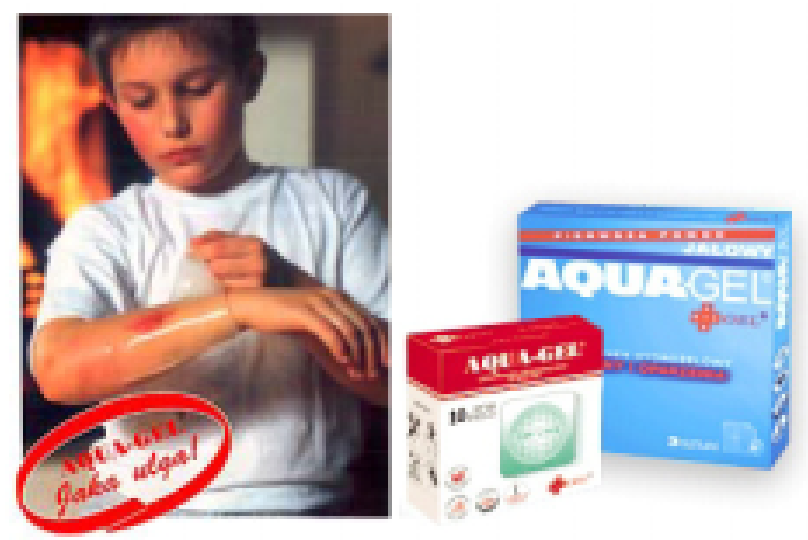

Figure 3. Commercial hydrogel wound dressing prepared by radiation processing [26].

Since the earliest of 1990s, the Center for Application of Isotope and Radiation Technology, National Nuclear Energy Agency (PATIR-BATAN) actively developed PVP/PVA based polymer hydrogel prepared by radiation for medical applications especially wound dressing. The hydrogel as shown in Figure 4 has characteristics for application as wound covering. Physico-chemical, mechanical, microbiological properties, toxicity and preclinical evaluations of this hydogel have been evaluated [22, 27-30]. The results of cytotoxicity test using human keratinocite (HaCat) cell line by dimethyl thiazolyl diphenyl tetrazolium bromide (MTT) assay in vitro show that these hydrogels are non toxic which is indicated from viability of HaCat 
cell for PVP based hydrogels as well as its constituents (glycerine, agar, honey and PEG) are higher than the control sample as shown in Figure 5. The hydrogel does not have allergic and sensitivity reactions to the skin. It is elastic, sterile, an efficient barrier for bacteria and also for excessive loss of body fluids, and allows the diffusion of oxygen towards the wound as well as is effective enough for treatment of open wound and infected burn wound. The hydrogel has several advantages over conventional dressing such as it high fluid absorption capability very suitable for exudates wound, is sterile, elastic, easy to change dressing without pain, prevents bacteria invasion from outside to the wound, is non antigenic and does not provoke allergic reaction.
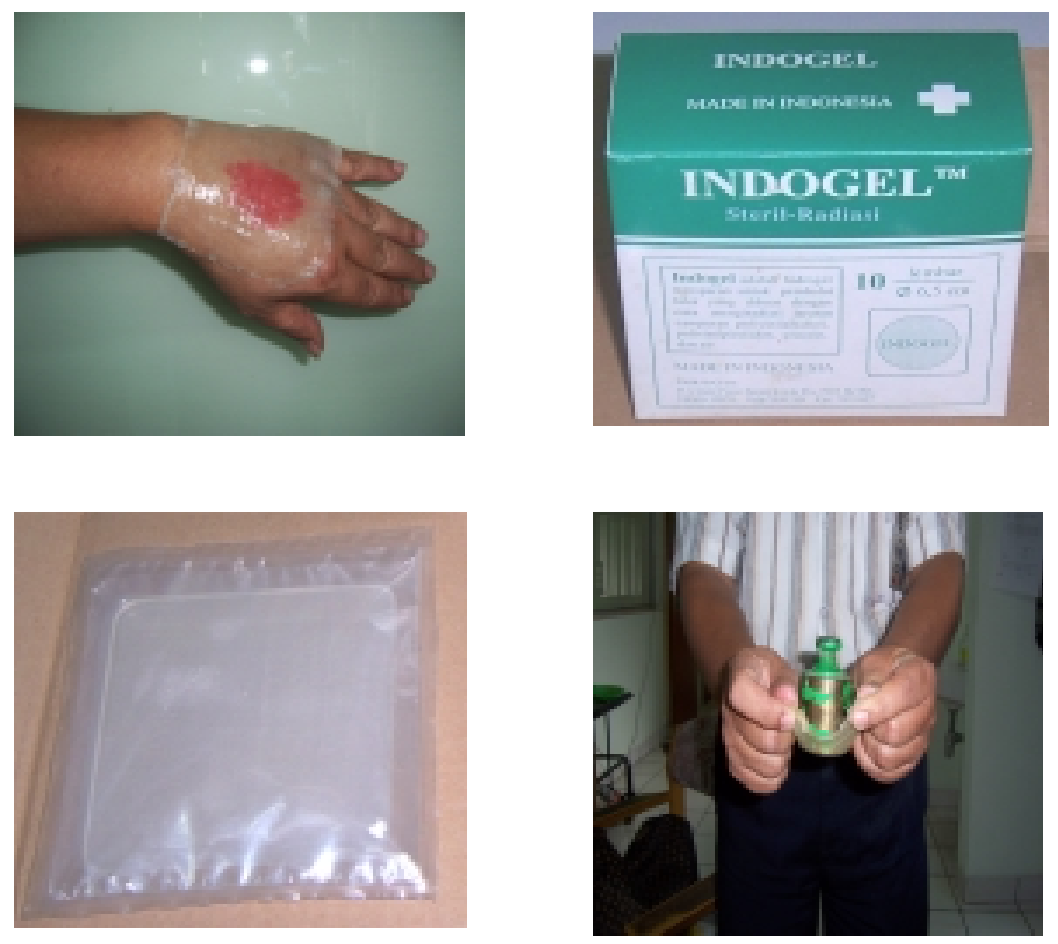

Figure 4. BATAN'S hydrogel wound dressing prepared by radiation technique. 


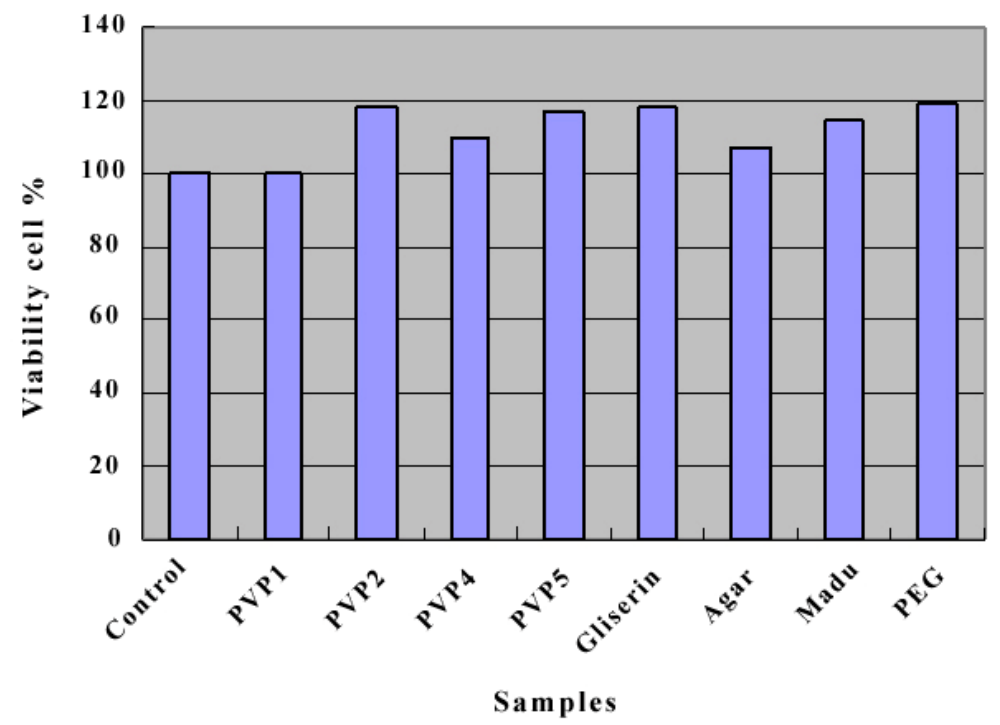

Figure 5. MTT assay results of PVP based hydrogel wound dressing for cytotoxicity analysis.

Beside of hydrogel wound dressing, we also develop hydrogel prepared by radiation for other purposes such as super water absorbent gel for application in diapers; hydrogel cooling pad, hydroxyapatite/polymer composite gel as synthetic bone graft for application in ortophaedics, periodontal and other bone defects [31]; implant materials such as synthetic discus nucleus and hydrogel GBR membrane for tissue engineering.

\section{Controlled Release of Drugs}

The aim in drug administration is to achieve a level of the agent at the target site such that its effectiveness is optimized. The periodic application by conventional techniques, such as orally or by intravenous injection, means that plasma level of the active agent will modulate about this optimal level. This runs the risk of achieving concentrations that are so high as to produce toxic side effects or so slow as to render the drug effectiveness. Poor patient compliance with the administration regime can exacerbate this problem [13]. Controlled release pharmaceuticals provide much more precise control over drug concentration in blood, and prolong drug activity [26]. Such drug delivery vehicles are generally constructed from synthetic polymeric materials into which the drug is incorporated, either by being sequestered within the polymer matrix or chemically bounded to the polymer backbone. Release of the agent can be achieved by having it diffuse out through the polymer matrix or having the device undergo some form of chemical erosion. 
The water-swollen hydrogels with high degrees of swelling can provide rate-controlling barriers to the diffusion of water-soluble drugs allowing the highest attainable fluxes from polymer. A new class of hydrogel which exhibits significant sensitivity to environmental changes such as temperature, $\mathrm{pH}$, electric field, ionic strength, salt type, solvent, external stress, and combination of these is called environmentally sensitive hydrogel [6]. Poly(N-isopropylacrylamide) is a thermo-sensitive hydrogels which has been widely studied. This gel is usually characterized by the lower critical solution temperature (LCST) of the backbone polymer or copolymer comprising the gel. When a hydrogel is composed of a lightly crosslinked matrix of an LCST polymer, the gel will shrink significantly over a narrow temperature range as the temperature is raised to the LCST or above as it can be seen in Fig. 6. Since the de-swelling occurs over a narrow temperature range, substances which have been previously adsorbed into the gel may release or deliver at this specific temperature.

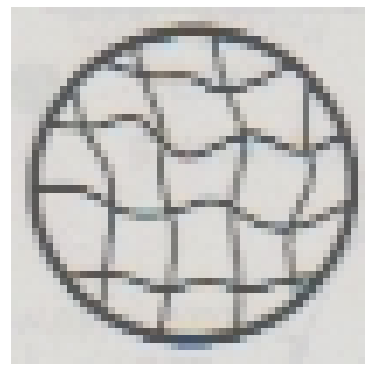

Low T

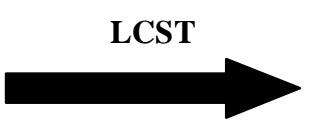

High $T$

(Swelling)

(Shrinking)

Figure 6. Illustration of $\mathrm{pH}$ sensitive hydrogel which has low critical solution temperature [6].

The use of biodegradable polymeric systems in controlled release drug delivery is desirable, since the dosage form will be degraded and eliminated from the body. This will avoid removal of the device from the body by surgery or other means when the device is no longer needed. Biodegradable polymeric systems also provide flexibility in the design of delivery system for large molecular weight drugs, such as proteins, which are not suitable for diffusion controlled release through nondegradable polymeric matrices [6].

\section{Other Applications}

Besides the above applications, hydrogels are continuously being subject of research for diagnostic devices which include catheters, electrode 
catheter, and carrier for enzyme immunoassay, cell culture substrate, tissue engineering and electrophoresis gels [32, 33].

\section{CONCLUSION}

It can be concluded that the role of radiation processing in preparation and production of hydrogel for medical applications is continuously growing. The main areas of application of hydrogels as biomaterials are synthetic wound covering and drug delivery system. Among various methods applied for the production of hydrogels, the radiation technique has many advantages, as it is a simple, efficient, clean and environmentally friendly process. It usually allows to combine the synthesis and sterilization in a single technological step, thus reducing costs and production time. Implants, drug delivery system, artificial organs, and bioengineering generally are domains in which radiation formed polymer materials begin to play an increasingly significant role.

\section{REFERENCES}

1. KROSCHWIT, Z., :"Polymers: Biomaterials and Medical Applications", Encyclopedia Reprint Series, John Wiley \& Sons, Inc., 52-60 (1989).

2. KOPECEK, J., Biomaterials, 7, 44-53 (2007).

3. KAZANSKII, K.S., and DUBROUSKII, S.A., :“Advance in Polymer Science”, Spring-Verlac, Berlin, 97-105 (1992).

4. HORAK, D., CERVINCA, M., and PUZA, V., Biomaterials, 18, 1335-1345 (1997).

5. SARIRI, R., Iranian polymer Journal, 6, 135-143 (1997).

6. PARK, K., SHALABY, S.W., and PARK, H., :"Biodegradable Hydrogels for Drug Delivery", Technomic Publishing Co. Inc., Lancaster, 6-21 (1993).

7. CHAPIRO, A., Radiat. Phys. Chem., 46, 159-171 (1995).

8. ROSIAK, J.M., ULANSKI, P., PAJEWSKI, L.A., YOSHII, F., and MAKUUCHI, K., Radiat. Phys. Chem., 46, 161-170 (1995).

9. SARAYDIN, D., KARADAG, E., CETINKAYA, S., and GUVEN, O., Radiat. Phys. Chem., 46, 1049-1058 (1995).

10. OKAZAKI, M., HAMADA, T., FUJ, H., MIZOBE, A., and MATSUZAWA, S., J. of Appl. Polym. Sci., 58, 2235-2246 (1995). 
11. HIRAI, M., HIRAI, T., SUKUMODA, A., NEMOTO, H., AMEMIYA, Y., KOBAYASHI, K., and UEKI, T., J. Chem. Soc. Faraday Trans., 91, 473-482 (1995).

12. HIRAI, T., NEMOTO, H., HIRAI, M., and HAYASHI, S., J. of Appl. Polym. Sci., 53, 79-88 (1994).

13. TIGHE, B.J., :"The encyclopedia of advanced materials", Elsevier Science Ltd., United Kingdom, Vol. 2, 1060-1069 (1994).

14. CHAPIRO, A., :"Radiation Chemistry of Polymeric Systems", Interscience Publisher New York, 339-360 (1962).

15. YAMAOKA, H., Radiation Chemistry of Polymer, UNDP/IAEA/RCA Regional Training Course on Radiation Chemistry, Japan (1991).

16. MILLER, A.A., LAWTON, E.J., and BALWIT, J.S., J. Polym. Sci., 14, 503-512 (1954).

17. CAREnZA, M., Radiat. Phys. Chem., 39, 485-495 (1992).

18. KUMAKURA, M., YOSHIDA.M., ASANO, M., and YAMADA, H., Radiat. Phys. Chem., 39, 577-586 (1992).

19. ROSIAK, J.M, OLEJNICZAK, J., and CHARLESBY, A., Radiat. Phys. Chem., 32, 691-702 (1988).

20. ROSIAK, J.M., ULANSKI, P., RIEZNICKI, A., Nucl. Inst. Method, B, 105, 335-346 (1995).

21. ROSIAK, J. M., J. Controlled Release, 31, 9-20 (1994).

22. DARWIS, D., YOSHII, F., MAKUUCHI, K., and RAZZAK, M.T., J. Appl. Polym. Sci., 55, 1619-1628 (1995).

23. YANG, J. M., and HSIU, G. H., J. Biomat. Res., 31, 281-290 (1996).

24. BEVER, M.B (Ed.), :"Encyclopedia of Material Science and Engineering”, Vol. 1, Pergamon Press, Oxford, 307-320 (1986).

25. REFOJO, M.F., :"Encyclopedia of Chemical Technology”, Vol. 6, John Wiley \& Sons Inc. New York, 720-735 (1979).

26. ROSIAK, J.M., :“Amer. Chem. Soc. Books, series 475”, Washington D.C., 271-295 (2001).

27. DARMAWAN, D., NAZLY, H., TATY, E., and LELY, H., Risalah Pertemuan Ilmiah Aplikasi Isotop dan Radiasi dalam Bidang Industri, Pertanian, dan Lingkungan, Jakarta, 14-15 Desember, 151-162 (1993).

28. DARMAWAN, D., LELY, H., TATY, E., RAHAYUNINGSIH, C, and NAZLY, H., Prosiding Seminar Nasional Himpunan Kimia Indonesia, Serpong, 324-332 (1999). 
29. RAZZAK, M.T., DARMAWAN, D., SUKIRNO, and ZAINUDDIN, Radiat. Phys. Chem, 62, 107-117 (2001).

30. DARMAWAN D., Jurnal Ilmiah Aplikasi Isotop dan Radiasi, 4, 53-61 (2008).

31. DARMAWAN D. dan YESSY W., Jurnal Ilmiah Aplikasi Isotop dan Radiasi, 4, 143-153 (2008).

32. KUDELA, V., :Hydrogels in Polymer Biomaterials and Medical Applications", John Wiley \& Sons, Inc., New York, 230-243 (1989).

33. DAVIT, J. T. H., and WHITTAKER, A. K., :"Encyclopedia of Polymer Science and Technology", John Wiley and Sons, 1-14 (2005). 